\title{
VOLUME MEASUREMENT OF SAND CARRIER USING UAV-BASED MAPPING
}

\author{
H. $\mathrm{He}^{1, *}, \mathrm{X} \cdot \mathrm{Xu}^{1}, \mathrm{~T} . \mathrm{Chen}^{2}, \mathrm{P} . \mathrm{Lu}^{3}$ \\ ${ }^{1}$ School of Geomatics, East China University of Technology, Nanchang 330013, China - (hehaiqing, 201810705035)@ecut.edu.cn \\ ${ }^{2}$ School of Water Resources \& Environmental Engineering, East China University of Technology, Nanchang 330013, China - \\ ct_201607@ecut.edu.cn \\ ${ }^{3}$ School of Geodesy and Geomatics, Wuhan University, Wuhan 430079, China - (lupeng1994@whu.edu.cn)
}

\section{Commission VI, WG VI/4}

KEY WORDS: Volume Measurement, Unmanned Aerial Vehicle (UAV), Point Cloud, Structure-from-Motion (SfM), Dense Matching

\begin{abstract}
:
To improve the operation efficiency and reduce the risks associated with exposing surveyors to danger during on-site operations, this paper presents a volume measurement approach of the sand carrier using unmanned aerial vehicle (UAV) imagery. Instead of the 3D surface interpolation of the sand carrier by total station measuring method or real-time kinematic global positioning system (RTK GPS) measuring method, the finely detailed surfaces of the sand carriers are reconstructed from dense point clouds derived by UAV-based mapping. Then, the volume of sand is calculated by the differential method, which multiplies the height difference between the UAV-derived 3D surfaces of the vessel and sand by the resolution of these surfaces. A total of 10 sand carriers are selected to test and evaluate the performance of the proposed approach, the absolute values of relative deviation between the volume obtained by UAV-based and the reference volume are approximately equal to $2 \%$, which can be considered acceptable and satisfy the need of volume measurement of sand carriers. Compared with the manual field measurement and the laser scanningbased method, the proposed approach performs better in the accuracy of volume measurement or the reliability under the circumstance of a moving carrier. The overall results suggest that UAV-mapping can be used as an effective alternative to the commonly used method for volume measurement of the sand carrier.
\end{abstract}

\section{INTRODUCTION}

Sand is an important raw material for many engineering projects and usually transported to the construction site by ship, the efficiency and accuracy of sand volume measurement play a key role in project management (Lu, 2011; Fawzy, 2015). At present, volume measurement of sand carrier is mainly manual field measurement, which is technically complex and timeconsuming because of the heavy workload, low efficiency, and empirical requirements. Also, this method is difficult to guarantee the accuracy of volume measurement.

Generally, based on the measuring instruments used, the traditional methods of volume measurement include total station measuring method and real-time kinematic global positioning system (RTK GPS) measuring method (Hamzah, 2011). Due to the limitations of viewpoint occlusion and the range of the sand carrier, the total station visibility requirements are difficult to meet. Besides, the sand carrier is difficult to keep stationary because it may often be disturbed by wind and water flow, in this case, the positions on the sand carrier may change greatly with time, which makes it difficult to be effective for time-consuming methods such as total station-based and RTK GPS-based methods. Besides, the common problem of the aforementioned measurements using traditional instruments is that they are not able to obtain sufficient three-dimensional (3D) object points to characterize the surface of the sand (Yilmaz, 2010; Bajtala, 2011). In recent years, 3D laser scanning and unmanned aerial vehicle (UAV)- based mapping have emerged as a promising techniques due to the ability to efficiently acquire a large amount of 3D point clouds and is widely used for volume measurement (Starek, 2013; Fan, 2015; Raeva, 2016; Xu, 2019). Especially, the backpack-type laser scanner with portability and flexibility is suitable to quickly measure the volume. However, the highprecision laser scanning instruments are costly and are not easily operated by surveyors in the narrow passages on the deck of the carrier. Even worse, the laser scanning may not be used to reconstruct the 3D surface of sand when the carrier is moving or shaking. Therefore, the laser scanning-based methods are not suitable for the volume measurement of sand carriers and are not popularized in this field. Recently, due to the ability to quickly obtain high-resolution remote sensing images and reconstruct high-precision 3D geometry structure, another high-tech UAV mapping technology has also been developed rapidly and broadly applied to the applications of agriculture, forestry, archaeology, resource investigation, environmental monitoring, 3D city modeling (Shahbazi, 2015; Mancini, 2017; Sluijs, 2018). In contrast to the laser scanning operation, UAV-mapping is low-cost and may offer the opportunity to measure the volume of sand under the circumstance of a moving or shaking carrier. That is, UAVmapping can provide a new way to suit this case of volume measurement of the sand carrier.

The aim of this paper is to assess the capabilities of UAVbased mapping for measuring the volume of sand carrier. A workflow for volume measurement based on low-cost UAV

\footnotetext{
* Corresponding author
} 
mapping including data acquisition and processing is proposed. This involves aerial triangulation with several ground control points and dense matching with semi-global algorithm (SGM), then high-precision and dense 3D points on the surface of sand can be derived from UAV overlapping images. Overall results confirm that UAV-based mapping can significantly improve the accuracy and efficiency of volume measurement.

\section{UAV MAPPING BASED SURFACE RECONSTRUCTION}

UAV mapping is highly integrated with the UAV platform, sensors, remote control, communication, navigation and positioning, and image processing techniques. The overlapping images can be acquired by the airborne camera and processed by photogrammetric software, thus the geospatial information of the ground objects can be derived. In this study, a low-cost small quadcopter, namely, DJI Phantom 4 PRO, which is used to capture the overlapping images, as shown in Figure 1. This UAV has a simple structure, flexible in operation, less preparation time, and low requirements for the landing site. It can take off and land at any time according to the mission needs and is not limited by data acquisition during the revisit period. Moreover, the UAV can directly acquire overlapping images of dangerous areas and areas beyond the reach of surveyors.

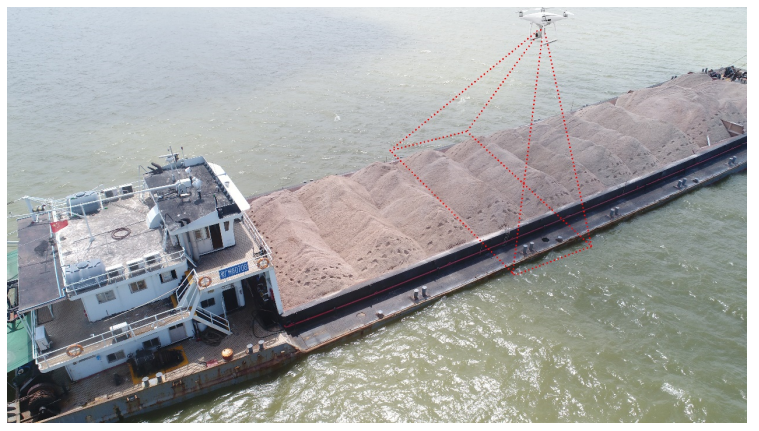

Figure 1. UAV data acquisition.

Similar to traditional aerial photogrammetry, UAV mapping also requires route planning according to the requirements of the mission, and then the overlapping images are acquired through the waypoints predefined. However, route planning is generally used to obtain images of stationary ground objects and not applicable for data acquisition under the circumstance of a moving or shaking object. Because of the small operating range, in this study, we manually operated the UAV to capture the overlapping images. As a consequence of this, the overlapping range between images is irregular. In addition, unlike the traditional aerial photogrammetry, initial exterior orientation parameters obtained from the low-cost airborne GPS and inertial measurement unit (GPS/IMU) are not available to assist aerial triangulation. Therefore, Structurefrom-motion $(\mathrm{SfM})$ is used to recover the 3D surface of the sand carrier due to the ability to allow 3D reconstruction from the overlapping but otherwise unordered images without a prerequisite of accurate GPS/INS data (He, 2019). To minimize the systematic errors of the airborne consumer-grade camera, which is pre-calibrated. The interior orientation parameters are then optimized through self-calibrating adjustment.

To satisfy the requirements of the volume measurement of the sand carrier, the aerial photography is conducted to acquire the overlapping images of the sand carrier before (no load) and after loading (full load). Note that the surface of the sand carrier only needs to be reconstructed once. The 3D reconstruction is achieved by a serial photogrammetric process, e.g., feature extraction and matching, SfM, dense matching. Then, the volume of sand is calculated based on the differential z-value between the two sets of point clouds with no load and full load. Specifically, the technical process is shown in Figure 2, which mainly includes ground control points (GCPs) placement, overlapping images acquisition, lens distortion correction, dense point clouds generation, and volume calculation etc.

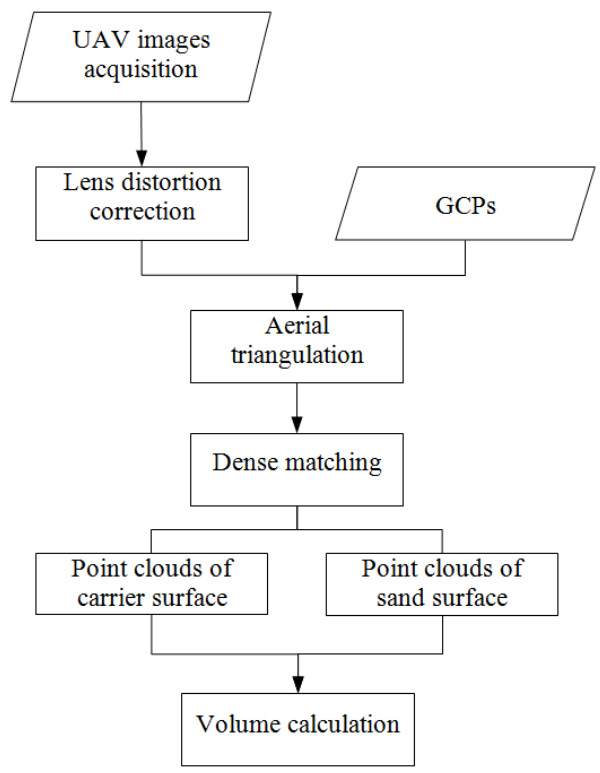

Figure 2. A technical workflow of UAV-mapping volume measurement of the sand carrier.

The volume measurement of this study still requires GCPs to georeference for absolute orientation. The difference with the common GCPs acquisition is that the GCPs are measured once on a static carrier and then used for absolute orientation regardless of whether the carrier is moving or not. In other words, this study ignores the background information in the overlapping images and only treats the carrier as a relatively stationary object. The GCPs are marked on the surface of the carrier, as illustrated in Figure 3.

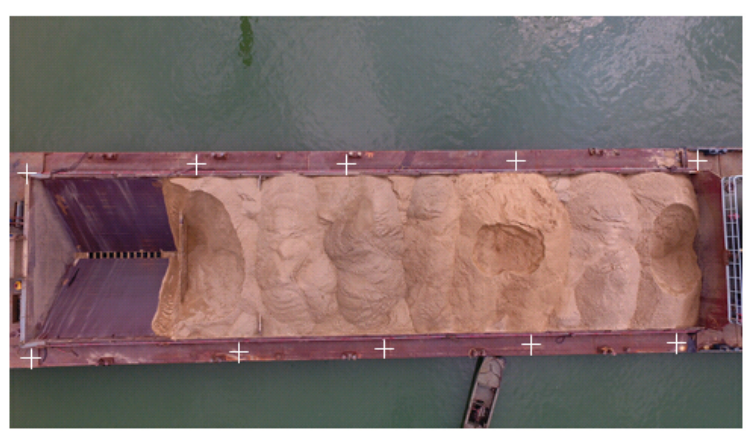

Figure 3. GCPs placements marked by white crosses.

The photogrammetric processes, e.g., feature extraction and matching, SfM, and dense matching (i.e., SGM), are done by using the previous studies (He, 2019). Then, the point clouds of carrier and sand surfaces are derived from UAV-based photogrammetry. It should be noted that the cost function used 
in the dense matching is slightly different from the SGM to ensure the matching reliability of repetitive or weak textures (shown in Figure 4) on the surface of the sand.
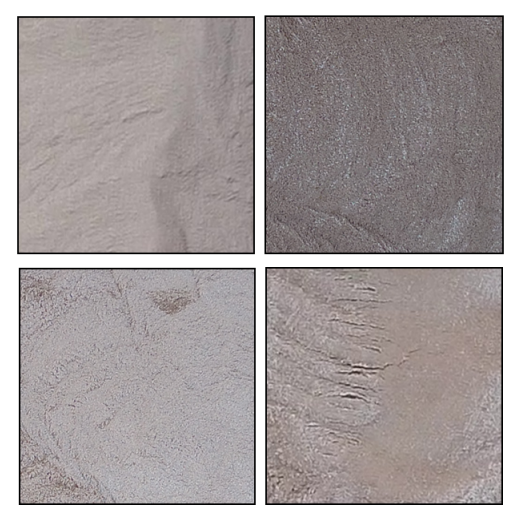

Figure 4. Four surfaces with repetitive or weak textures of the sand exhibited as examples.

Based on the SGM algorithm (Hirschmuller, 2005), let $L_{r}$ denote a cost path that traverses in the direction $r$. The minimum cost path $L_{r}\left(p_{x}, d\right)$ for the pixel $p_{x}$ at disparity $d$ can be defined recursively as

$$
L_{r}\left(p_{x}, d\right)=C\left(p_{x}, d\right)+\min \left(\begin{array}{l}
L_{r}\left(p_{x}-r, d\right), \\
L_{r}\left(p_{x}-r, d-1\right)+P_{1}, \\
L_{r}\left(p_{x}-r, d+1\right)+P_{1}, \\
\min _{i} L_{r}\left(p_{x}-r, i\right)+P_{2}, \\
\operatorname{dist}\left(p_{x}-r, p_{x}\right)
\end{array}\right)
$$

where the pixel-wise matching cost $C\left(p_{x}, d\right)$ can be calculated based on mutual information (MI) (Viola, 1995). $\operatorname{dist}(\cdot)$ denotes an operation of gradient Euclidean distance and is enforced to establish a more robust similarity measure than the original cost function, the gradient calculation is similar to the operation used in the scale-invariant feature transform (SIFT) algorithm.

\section{VOLUME CALCULATION}

The dense point clouds obtained from UAV-mapping are split into a series of grids with a size of $d \times d$ on the $x o y$ coordinate plane, which is shown in Figure 5. The z-value of each grid is defined as an average value of $3 \mathrm{D}$ points within the grid. Then, the mathematical differential method is used to calculate the volume $V$ of the sand carrier by multiplying the z-value difference between the sand and carrier surface models. And the differential calculation can be defined as

$$
V=\sum_{i=1}^{M} \sum_{j=1}^{N}\left(Z_{i, j}^{\mathrm{sand}}-Z_{i, j}^{\mathrm{carrier}}\right) \cdot d_{i, j}^{2}
$$

where $M$ and $N$ are the rows and columns of the grids, respectively; $Z^{\text {sand }}$ and $Z^{\text {carrier }}$ are the z-values on the surface of sand and vessel, correspondingly.

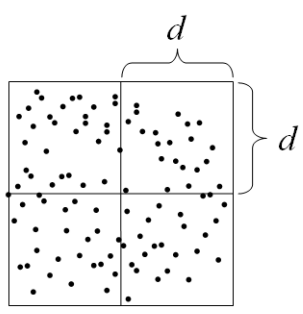

(a)

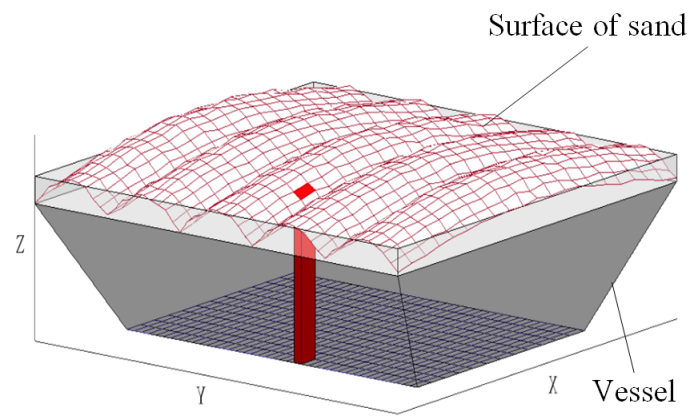

(b)

Figure 5. Differential method for volume measurement. (a) denotes the grids of with a size of $d \times d$. (b) represents the illustration of the differential method.

\section{EXPERIMENTS AND DISCUSSION}

The experimental area is located in the Pearl River, where sand carriers frequently pass through. The UAV used for data acquisition is a consumer-grade UAV, i.e., DJI Phantom 4 PRO shown in Figure 6, with 20-megapixel RGB true color obtained from 1-inch complementary metal-oxide-semiconductor (CMOS) sensor. Besides, the DJI Phantom 4 PRO has an integrated remote control with a 5.5-inch $1080 \mathrm{p}$ display that allows the operator to view the images captured by the drone in real-time and adjust the flight status through the highresolution image transmission system. Other related parameters of the DJI Phantom 4 PRO are given in Table 1 (DJI, 2016), thus the UAV image acquisitions are performed under the requirements of the parameters. Because the width of the sand carrier is small, it can be photographed with a single strip, and the overlaps are set to $80 \%$. The flight height is adjusted to ensure that the width of the carrier is no less than $60 \%$ of the image. Besides, the airborne camera is calibrated using the 2D chessboard pattern, four views are exhibited in Figure 7, and the initial camera parameters are given in Table 2.

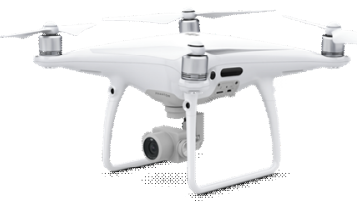

Figure 6. DJI Phantom 4 PRO.

\begin{tabular}{cccc}
\hline Weight & $\begin{array}{c}\text { Flight } \\
\text { time }\end{array}$ & $\begin{array}{c}\text { Flight } \\
\text { altitude }\end{array}$ & $\begin{array}{c}\text { Withstand } \\
\text { wind }\end{array}$ \\
\hline $1.388 \mathrm{~kg}$ & $\leq 30 \mathrm{~min}$ & $\leq 6000 \mathrm{~m}$ & $\leq 10 \mathrm{~m} / \mathrm{s}$ \\
\hline
\end{tabular}

Table 1. Several parameters of DJI Phantom 4 PRO. 


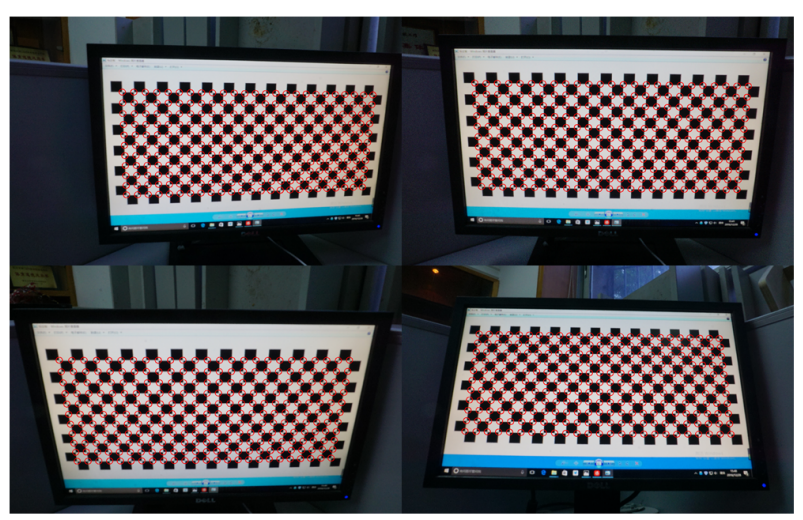

Figure 7. Four views of the $2 \mathrm{D}$ chessboard exhibited as examples.

\begin{tabular}{|c|c|}
\hline Focus $f x:$ & 3960.9648 \\
\hline Focus $f y:$ & 3959.1806 \\
\hline Principal Point $x:$ & 2977.5390 \\
\hline
\end{tabular}

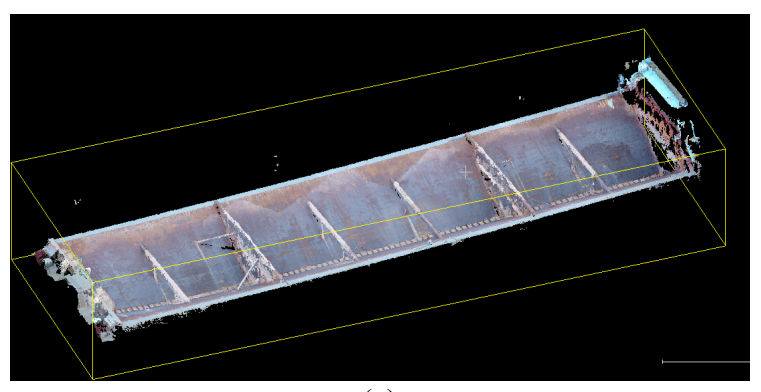

(a)

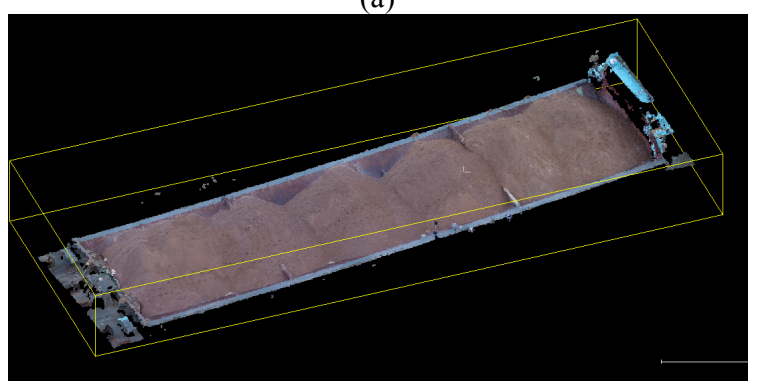

(c)

\begin{tabular}{|c|c|}
\hline Principal Point $y:$ & 1946.4755 \\
\hline Radial Distortion $k 1:$ & -0.048182446509599686 \\
\hline Radial Distortion $k 2:$ & 0.040056683123111725 \\
\hline Tangential Distortion $p 1:$ & -0.003868975676596164 \\
\hline Tangential Distortion $p 2:$ & -0.001025215955451131 \\
\hline
\end{tabular}

Table 2. Parameters of the airborne camera.

Following the aforementioned requirements, the DJI Phantom 4 PRO is operated to capture the carrier before and after loading the sand in the experimental area. A total of 10 carriers (half stationary carriers No. 1-5 and a half moving carriers No. 6-10 shown in Table 2) are photographed in the cases of noload and full load. Figure 8 shows the dense point clouds and the depth maps of an example derived by the SfM and SGM algorithms, the visualization results show that the finely detailed surfaces of vessel and sand are reconstructed.

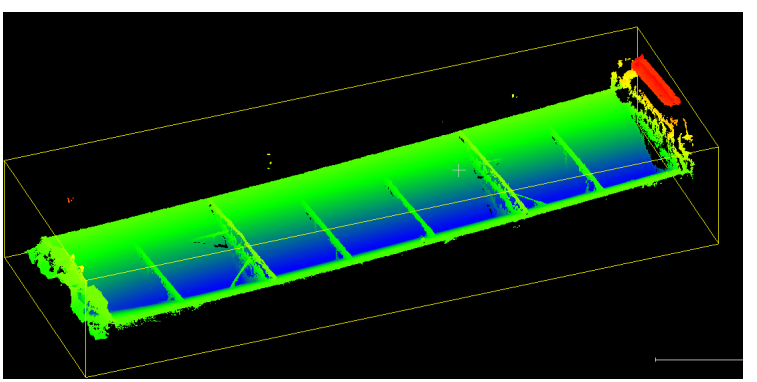

(b)

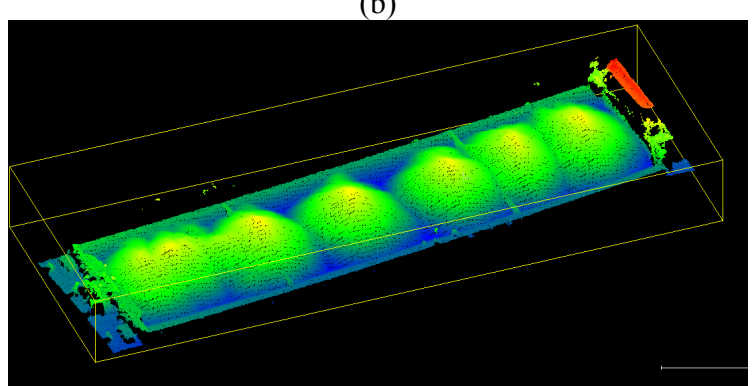

(d)

Figure 8. Results of 3D reconstruction. (a) and (c) represent the dense point clouds rendered in RGB true color of vessel and sand, respectively. (b) and (d) represent the depth maps of vessel and sand, respectively.

\begin{tabular}{|c|c|c|c|c|c|c|c|c|}
\hline \multirow[b]{2}{*}{ No. } & \multirow{2}{*}{$\begin{array}{c}\text { Image } \\
\text { numbe } \\
\mathbf{r} \\
\text { (UAV) }\end{array}$} & \multicolumn{4}{|c|}{ Volume $\left(\mathrm{m}^{3}\right)$} & \multicolumn{3}{|c|}{ Deviation $\sigma$} \\
\hline & & $\begin{array}{c}\text { Referenc } \\
\text { e }\end{array}$ & $\begin{array}{c}\text { Manua } \\
\mathbf{l}\end{array}$ & $\begin{array}{c}\text { Laser } \\
\text { scanning- } \\
\text { based }\end{array}$ & $\begin{array}{l}\text { UAV- } \\
\text { based }\end{array}$ & $\begin{array}{c}\text { Manua } \\
1\end{array}$ & $\begin{array}{c}\text { Laser } \\
\text { scanning- } \\
\text { based }\end{array}$ & UAV-based \\
\hline 1 & 21 & 2977 & 3298 & 2942 & 2927 & $10.78 \%$ & $-1.18 \%$ & $-1.68 \%$ \\
\hline 2 & 19 & 2813 & 3017 & 2853 & 2841 & $7.25 \%$ & $1.42 \%$ & $1.00 \%$ \\
\hline 3 & 19 & 2795 & 3103 & 2857 & 2861 & $11.02 \%$ & $2.22 \%$ & $2.36 \%$ \\
\hline 4 & 18 & 2644 & 2981 & 2593 & 2673 & $12.75 \%$ & $-1.93 \%$ & $1.10 \%$ \\
\hline 5 & 19 & 2710 & 2809 & 2756 & 2753 & $3.65 \%$ & $1.70 \%$ & $1.59 \%$ \\
\hline 6 & 18 & 2392 & 2473 & - & 2356 & $3.39 \%$ & - & $-1.51 \%$ \\
\hline 7 & 19 & 2677 & 2957 & 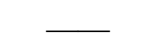 & 2711 & $10.46 \%$ & - & $1.27 \%$ \\
\hline 8 & 18 & 2497 & 2636 & - & 2543 & $5.57 \%$ & - & $1.84 \%$ \\
\hline 9 & 20 & 2988 & 2704 & - & 2973 & $-9.50 \%$ & - & $-0.50 \%$ \\
\hline 10 & 19 & 2529 & 2742 & - & 2564 & $8.42 \%$ & 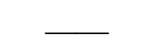 & $1.38 \%$ \\
\hline
\end{tabular}

Table 3. Comparisons of volume calculation using manual field measurement (Manual), laser scanning based and UAV-based. methods. 
Table 3 shows the results of volume measurement using the proposed approach, which is also compared with other methods, i.e., manual field measurement and laser scanning based methods. The relative deviation $\sigma$ of volume measurement is defined as

$$
\sigma=\frac{\left(V_{\mathrm{M} / \mathrm{L} / \mathrm{U}}-V\right)}{V} \cdot 100 \%,
$$

in which $V$ denotes the reference volume, $V_{\mathrm{M} / \mathrm{L} / \mathrm{U}}$ represents one of the volumes obtained by manual field measurement, laser scanning-based, and UAV-based methods.

Note that the reference volume is determined by the same sand with a regular shape such as trapezoid, which is shaped on land shown in Figure 11a. Then, the volume of sand is calculated by the mathematical formula of a trapezoid. Similar to the acquisition of the true value, the sand in the carrier is generally shaped into trapezoid (shown in Figure 11b) in the manual field measurement, and then the measuring tape is used to measure the size of the trapezoid for volume calculation (Bajtala, 2012). For the backpack-type laser scanning-based method, a Velodyne LiDAR with HDL-32E LiDAR sensor shown in Figure 9 is used to obtain the point clouds of the surfaces of vessel and sand, as shown in Figure 11c.

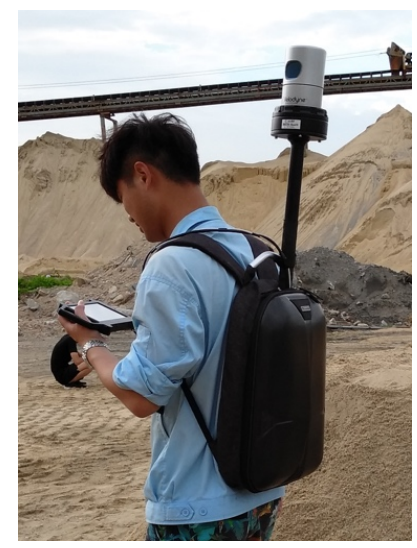

Figure 9. Backpack-type laser scanning instrument.

As can be seen from Table 2, the volumes calculated by the manual measurement have a large deviation from the reference values, some of which are more than $10 \%$. It indicates that the reliability of this manual method is poor due to the difficulty of quality control of trapezoid. In the first five results, the total deviation of the laser scanning-based method is similar to the

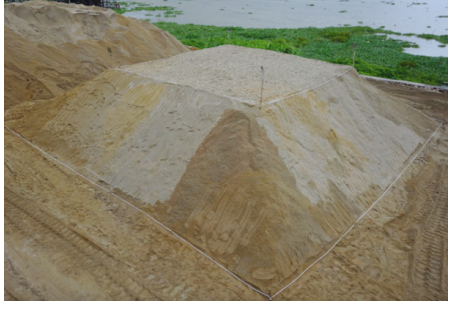

(a)

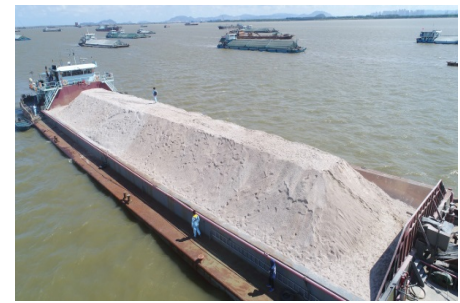

(b)
UAV-based method obtained, and the absolute values of relative deviation are relatively small and approximately equal to $2 \%$. In contrast, both of the laser scanning-based and UAVbased methods are significantly better than the manual measurement, the deviation can satisfy the requirements of sand carrier measurement (Raeva , 2016; Abbaszadeh, 2017). However, the moving carrier may cause laser sensor vibration and generate invalid data, e.g., No. 6-10.

Besides, the results obtained by the dense matching of the proposed cost function are compared with that of the original SGM cost function. The statistical results of 10 sand carriers are shown in Figure 10, it can be seen that the proposed cost function performs better in terms of smaller relative deviations. It should be noted here that we did not use GCPs to evaluate the accuracy of surface reconstruction, because the sand surface is unstable and soft, and the measuring instrument placed on the sand may cause deformation of the sand surface. Besides, on the one hand, the UAV-based method can effectively avoid the influence of site obstacles without the need of boarding, the whole operation process is simple, fast and safe, and the data is presented in the form of image, which is highly intuitive; on the other hand, the DJI Phantom 4 PRO is low-cost, and there is no need for multiple operators to cooperate, which also saves manpower and financial resources. Thus, results suggest that the UAV-based method can be used as an effective alternative to the manual field measurement for volume calculation of sand carrier.

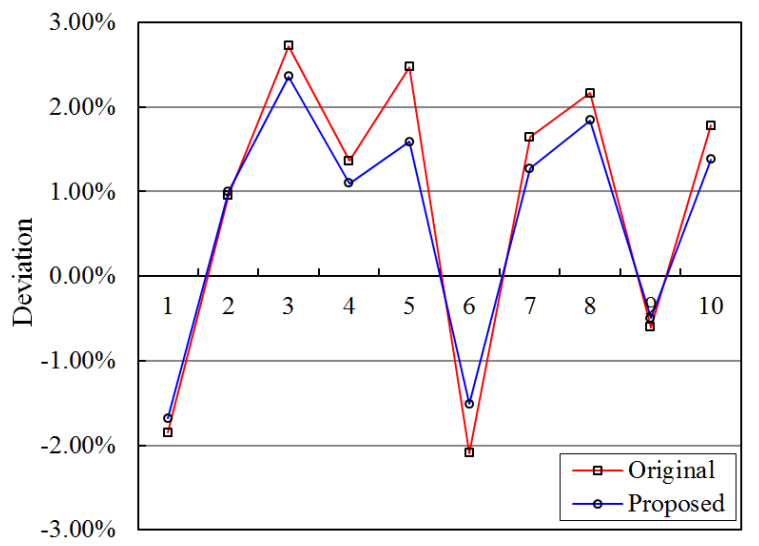

Figure 10. Comparisons of the relative deviation obtained by the original and proposed cost functions.

Figure 11. Volume measurements of other three methods. 


\section{CONCLUSIONS}

This paper presents the data acquisition and processing of volume measurement of sand carriers based on UAV mapping. Briefly, a volume measurement is performed by the differential method with the height difference between the UAV-derived dense point clouds of vessel and sand. Compared with the manual field measurement and laser scanning-based method, the following conclusions are drawn:

(1) The volume measured by the manual field method is poor and unreliable, while the laser scanning-based and UAV-based methods can obtain results close to the reference volume. However, the laser scanning-based method maybe not available to measure the volume under the circumstance of a moving carrier.

(2) Based on the advantages of UAV aerial photography, data acquisition can be completed without the need of surveyors boarding a carrier, which improves the operation efficiency and reduces the risks associated with exposing surveyors to danger during on-site operations. Besides, the UAV instrument is lowcost and easily operated, which is conducive to popularize the application of UAV-based volume measurement.

(3) The high-precision dense point clouds derived from UAVmapping can characterize the finely detailed surface of the vessel and sand. The deviation between the volume obtained by UAV-based and the reference volume is approximately equal to $2 \%$, thus the UAV-based method can be used as an effective alternative to the manual field measurement for volume calculation of sand carrier.

\section{ACKNOWLEDGEMENTS}

This work was financially supported by the National Natural Science Foundation of China (Grant No.41861062, No.41401526) and the Natural Science Foundation of Jiangxi Province of China (Grant No.20171BAB213025, No.20181BAB203022).

\section{REFERENCES}

Lu, T-F., Myo, M.T.R., 2011: Optimal Stockpile Voxel Identification Based on Reclaimer Minimum Movement for Target Grade. International Journal of Mineral Processing 98(1-2), 74-81.

Fawzy, H.E-D., 2015: The Accuracy of Determining the Volumes Using Close Range Photogrammetry. IOSR Journal of Mechanical and Civil Engineering 12(2), 10-15.

Hamzah, H.B., Said, S.M., 2011: Measuring Volume of Stockpile Using Imaging Station. Geoinformation Science Journal 11(1), 15-32.

Yilmaz, H.M., 2010: Close Range Photogrammetry in Volume Computing. Experimental Techniques 34(1), 48-54.

Bajtala, M., Brunčák, P., Kubinec, J., Lipták, M., Sokol, Š., 2011: The Problem of Determining the Volumes Using Modern Surveying Techniques. Ad Alta Journal of Interdisciplinary Research 01(02), 147-150.

Starek, M.J., Mitasova, H., Wegmann, K.W., Lyons, N., 2013: Space-Time Cube Representation of Stream Bank Evolution
Mapped by Terrestrial Laser Scanning. IEEE Geoscience and Remote Sensing Letters 10(6), 1369-1373.

Fan, L., Atkinson, P.M., 2015: Accuracy of Digital Elevation Models Derived From Terrestrial Laser Scanning Data. IEEE Geoscience and Remote Sensing Letters 12(9), 1923-1927.

Raeva, P.L., Filipova, S.L., Filipov, D.G., 2016: Volume computation of a stockpile - a study case comparing gps and uav measurements in an open pit quarry. Int. Arch. Photogramm. Remote Sens. Spatial Inf. Sci., XLI-B1, 999-1004.

Sayab, M., Aerden, D., Paananen, M., Saarela, P., 2018: Virtual Structural Analysis of Jokisivu Open Pit Using 'Structure-from-Motion' Unmanned Aerial Vehicles (UAV) Photogrammetry: Implications for Structurally-Controlled Gold Deposits in Southwest Finland. Remote Sensing 10(8), 1296.

Xu, Z., Xu, E., Wu, L., Liu, S., Mao, Y., 2019: Registration of Terrestrial Laser Scanning Surveys Using Terrain-Invariant Regions for Measuring Exploitative Volumes over Open-Pit Mines. Remote Sensing 11(6), 606.

Shahbazi, M., Sohn, G., Théau, J., Menard, P., 2015 Development and Evaluation of a UAV-Photogrammetry System for Precise 3D Environmental Modeling. Sensors 15(11), 27493-27524.

Mancini, F., Castagnetti, C., Rossi, P., Dubbini, M., Fazio, N.L., Perrotti, M., Lollino, P., 2017: An Integrated Procedure to Assess the Stability of Coastal Rocky Cliffs: From UAV Close-Range Photogrammetry to Geomechanical Finite Element Modeling. Remote Sensing 9(12), 1235.

Sluijs, J.V., Kokelj, S.V., Fraser, R.H., Tunnicliffe, J., Lacelle, D., 2018: Permafrost Terrain Dynamics and Infrastructure Impacts Revealed by UAV Photogrammetry and Thermal Imaging. Remote Sensing 10(11), 1734.

He, H., Yan, Y., Chen, T., Cheng, P., 2019: Tree Height Estimation of Forest Plantation in Mountainous Terrain from Bare-Earth Points Using a DoG-Coupled Radial Basis Function Neural Network. Remote Sensing 11(11), 1271.

Hirschmuller, H., 2005: Accurate and efficient stereo processing by semi-global matching and mutual information. IEEE Computer Society Conference on Computer Vision and Pattern Recognition (CVPR'05), San Diego, CA, USA, 807814.

Viola, P., Wells, W.M., 1995: Alignment by maximization of mutual information. Proceedings of IEEE International Conference on Computer Vision, Cambridge, MA, USA, 24, $137-154$.

DJI, 2016: Phantom 4 Pro/Pro+ User Manual. https://dl.djicdn.com/downloads/phantom_4 pro/Phantom $+4+$ Pro + Pro + Plus + User + Manual +v1.0.pdf.

Abbaszadeh, S., Rastiveis, H., 2017: A comparison of closerange photogrammetry using a non-professional camera with field surveying for volume estimation. Int. Arch. Photogramm. Remote Sens. Spatial Inf. Sci., XLII-4/W4, 1-4. 